\title{
Analysis of power flow under non-sinusoidal conditions in the presence of harmonics and interharmonics using geometric algebra
}

\author{
Francisco G. Montoya ${ }^{\mathrm{a}}$, Raúl Baños ${ }^{\mathrm{a}}$, Alfredo Alcayde ${ }^{\mathrm{a}}$, Francisco M. Arrabal-Campos ${ }^{\mathrm{a}}$ \\ ${ }^{a}$ CeiA3, Department of Engineering, University of Almería, Carretera de Sacramento s/n, 04120 Almería (Spain)
}

\begin{abstract}
The calculation of power flow in power systems with the presence of harmonics has been properly studied in the scientific literature. However, power flow calculation considering interharmonic components is still an open question. Traditional methods based on the IEEE1459 standard have proven to be valid and accurate only for linear and sinusoidal systems, but have been criticized for non-linear and non-sinusoidal systems because they are not able to explain correctly the current and voltage interactions beyond the active power. This paper proposes the use of a novel mathematical framework called geometric algebra (GA) to study the power flow considering the interaction of current and voltage harmonics and interharmonics. The use of GA enables the precise determination of the direction and magnitude of the total and single active power flow for each component, as well as other power elements related to the non-active power due to cross interaction. Moreover, this paper makes a novel contribution to the definition of interharmonics in geometric algebra space that has not been done before. To test the validity of the method, both linear and nonlinear circuits are proposed and solved by applying voltages and currents with harmonic and interharmonic components. The results obtained show that power flow can be analyzed under the prism of the principle of energy conservation ( $\mathrm{PoCoE})$ in a way that allows a better understanding of the power spectrum due to the interaction of harmonics and interharmonics of voltage and current.
\end{abstract}

Keywords: Power flow, Harmonics, Interharmonics, Non-sinusoidal conditions, Geometric Algebra.

\section{Introduction}

The proliferation of electronic devices has increasingly led to poor power quality in the electricity supply [1]. One of the most commonly used parameters for measuring power quality is the waveform distortion of voltage and current [2]. This waveform can be affected by the presence of spectral frecuencies other than the

Email addresses: pagilm@ual.es (Francisco G. Montoya), rbanos@ual.es (Raúl Baños), aalcayde@ual.es (Alfredo Alcayde), fmarrabal@ual.es (Francisco M. Arrabal-Campos) 
$50 / 60 \mathrm{~Hz}$ fundamental component. These spectral components can be integer multiples of the fundamental (harmonics) or not (interharmonics), depending on various factors such as the type of installation, receivers or class of supply. If they are not integer multiples, IEC 61000-2-1 defines them precisely as "Between the harmonics of the power frequency voltage and current, further frequencies can be observed which are not an integer of the fundamental. They can appear as discrete frequencies or as a wide-band spectrum" [3]. A particular case of interharmonics are the so-called subharmonics, i.e. spectral components with a frequency lower than the fundamental and which is not an integer multiple of it. The main causes for the appearance of interharmonics are usually two: on the one hand, the random change in the magnitude of the value of the voltage or current or the phase angle causes the appearance of intermediate spectral effects such as picket fence or spectral leakage that lead to the generation of fake interharmonic; on the other hand, the massive use of non-linear loads, and especially those that interact with true interharmonic power systems, such as ship propulsion systems, power inverters between different frequency systems, wind turbines, etc [4]. This article will focus on the study of true interharmonics, i.e. interharmonics of known origin caused by real devices.

Despite the clear interest in power quality [5], and the large number of studies of the influence of harmonics on power grids, there has not been much attention paid to the study of interharmonics, so it is necessary to look deeper into the causes that provoke them, as well as the consequences they have $[6,7]$. Currently, they are not a real or compelling problem, but it is expected that in the future they may become so, therefore it is necessary to pay attention to this problem [8]. For example, Ravindran and Bollen [9] investigates the existence of interharmonic emissions from an MPPT driven grid-connected PV inverter to identify their severity and persistence. Kim et al. [10] also talk about the impact of interharmonics on offshore electrical systems and how interaction with other harmonics can cause serious vibration problems that cause systems to shut down. For this, it is essential to use powerful and wellfounded mathematical circuit theory analysis tools. So far, the main analysis tool has been the application of Fourier analysis, and its FFT algorithmic implementation [4]. Other techniques such as wavelets also make an interesting contribution [11] when studying the interaction between voltage and current so that power flows can be clearly established. Kim et al. [10] propose the use of the cross power spectrum technique for interharmonic power analysis to determine real and reactive power flows. On the other hand, Petrovic [12] reviews undersampling methods for voltage and current signals with both harmonics and interharmonics in order to obtain active power measurements.

Geometric Algebra or Clifford Algebra has successfully been used in several scientific disciplines such as 
engineering, physics or computer science achieving excellent results $[13,14]$. In particular, it has proven to be a powerful and flexible tool for representing the flow of energy and power in electrical systems [15, 16]. Some researchers have proposed the use of Clifford's algebra as a mathematical tool to address the multicomponent nature of power in non-sinusoidal contexts $[17,18,19]$. The concept of non-active, reactive or distorted power acquires a meaning that is more in line with its mathematical significance, making it possible to better understand energy balances and to verify the principle of energy conservation. The concept of multi-component power within the scope of geometric algebra [20] is used in this article to demonstrate its feasibility for determining the net power flow in a non-sinusoidal electrical circuit, the direction and sense of such power, as well as its use for calculating the geometric or net power factor defined as the ratio between the active power and the norm of the multivector power. This paper also proposes a new extension to CastroNúñez's theory $[15,16,20]$, which has not been published previously, so that it takes into consideration the coding of interharmonics in space $\mathcal{G}_{\mathcal{N}}$. Thereby, it is possible to conveniently study the interactions between fundamental, harmonic and interharmonic components leading to different power components, while taking into account their senses, i.e. whether they are powers flowing from the source to the load or vice versa. Some specific applications in power systems have been already found, such as passive filtering [21]. In order to validate the theory presented in this paper, three cases of study are proposed where the circuit theory based on GA is applied to linear and non-linear loads.

The rest of the paper is structured as follows: Section 2 summarizes the theoretical basis of interharmonics; Section 3 introduces the analysis of electrical circuits by GA and the new formulation applied to interharmonics; Section 4 presents the results obtained in three case studies; Section 5 presents the main conclusions of this work.

\section{Interharmonic basics}

The harmonic component concept emerges from the application of Fourier analysis to periodic time signals. Through this technique, it is possible to reconstruct any periodic signal based on the sum of sinetype components. For example, if you have a voltage signal $v(t)$ (see Figure 1) that is periodic with period $T$, it is possible to perform the Fourier series decomposition according to

$$
v(t)=a_{0}+\sum_{n=1}^{\infty} a_{n} \cos n \omega t+\sum_{n=1}^{\infty} b_{n} \sin n \omega t=\sum_{n=0}^{\infty} c_{n} e^{j n \omega t}
$$

where $\omega=\frac{2 \pi}{T}, n$ is an integer and the coefficients are 


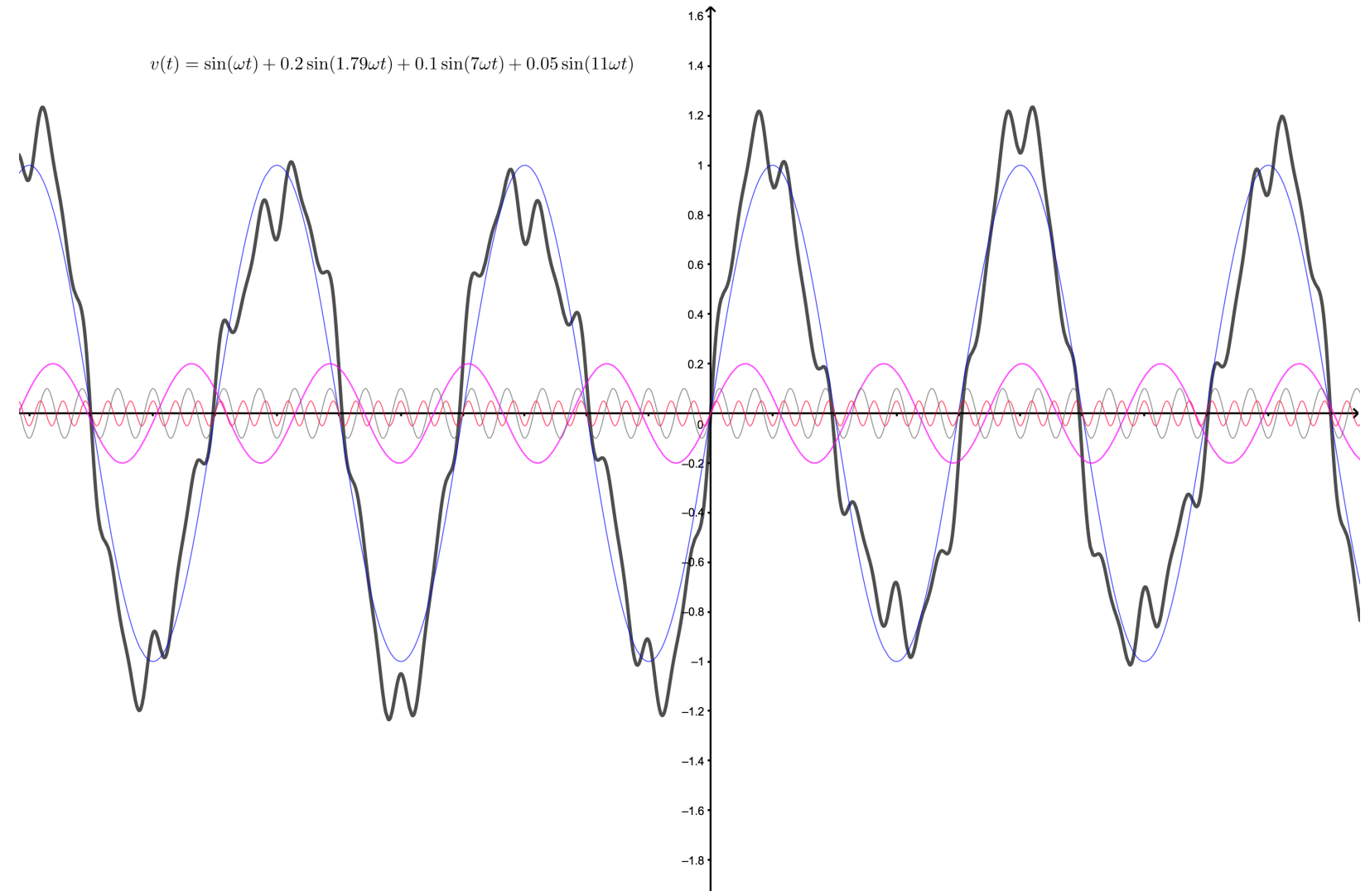

Figure 1: Power signal waveform with harmonics and interharmonics 


$$
\begin{aligned}
a_{0} & =\frac{1}{T} \int_{0}^{T} v(t) d t \\
a_{n} & =\frac{2}{T} \int_{0}^{T} v(t) \cos (n \omega t) d t \\
b_{n} & =\frac{2}{T} \int_{0}^{T} v(t) \sin (n \omega t) d t \\
c_{n} & =\frac{1}{2}\left(a_{n}-j b_{n}\right)=\frac{1}{T} \int_{0}^{T} v(t) e^{-j n \omega t} d t
\end{aligned}
$$

The discrete implementation of the Fourier Transform in the digital domain is based on the Discrete Fourier Transform (DFT):

$$
X\left(f_{k}\right)=\frac{1}{N} \sum_{n=0}^{N-1} x\left(t_{n}\right) e^{-j 2 \pi k n / N}
$$

where $f_{k}=\frac{k}{T_{s} N}=\frac{k}{T}$. The above equation carries the implicit assumption that the signal is repeated every $N$ samples, i.e. it contains a period $T$. If a higher number of samples is chosen, so that there are $p$ periods contained in the $N$ samples, then the spectral resolution will increase according to the expresión

$$
\Delta f=\frac{1}{p T}
$$

Thus, it is possible to increase the precision when it comes to detecting the possible interharmonics that may exist in a signal, whatever its origin is (real or fake). For example, for a fundamental frequency signal of $50 \mathrm{~Hz}$, you can choose a sample length equivalent to 10 periods $T$, so the frequency resolution would be $\Delta f=50 / 10=5 \mathrm{~Hz}$. If there are interharmonics multiple of $5 \mathrm{~Hz}$, they can be detected by the DFT. Naturally, the greater the number of samples, the more options there are to capture interharmonics precisely, but with the added cost of finding variations in the amplitude of the signal, which will inexcusably cause the appearance of other interharmonics that are not due to phenomena typical of electrical equipment. In any case, the application of the DFT allows us to obtain the coefficients of both harmonics and interharmonics to represent a voltage or current signal in the frequency domain. Thanks to these coefficients, in the next section it will be demonstrated the possibility of carrying out a transformation to the geometric space where it is possible to operate and obtain the power flows that arise as a result of the interaction between the different harmonics and interharmonics. 


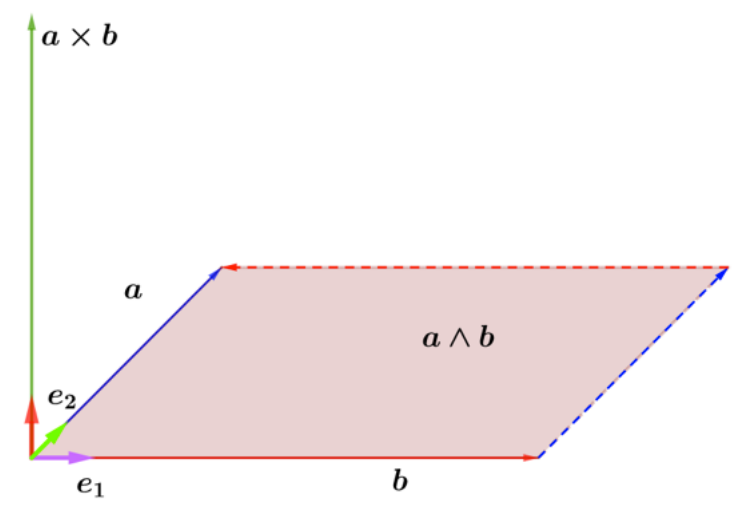

Figure 2: Representation of a bivector $\boldsymbol{a} \wedge \boldsymbol{b}$. Note that the classical vectorial product $\boldsymbol{a} \times \boldsymbol{b}$ is also represented as the perpendicular vector to plane formed by $\boldsymbol{a}$ and $\boldsymbol{b}$.

\section{Geometric algebra applied to harmonics and interharmonics}

Geometric algebra has its origins in the work of Clifford and Grassman in the 19th century. Unfortunately, their theory did not receive so much attention until its impulse by Hestenes and others [13, 14, 22]. Traditional concepts such as vector, spinor, complex numbers or quaternions are naturally explained as members of subspaces in GA. It can be easily extended in any number of dimensions, being this one of its main strengths. Because these are geometric objects, they all have direction, sense and magnitude. The basic concepts are widely explained in $[23,21,16]$ but the most important are reproduced here for the sake of completeness. One of the core concepts in GA is the geometric product between vectors $\boldsymbol{a}=\alpha_{1} \boldsymbol{e}_{1}+\beta_{1} \boldsymbol{e}_{\mathbf{2}}$ and $\boldsymbol{b}=\alpha_{2} \boldsymbol{e}_{\mathbf{1}}+\beta_{2} \boldsymbol{e}_{\mathbf{2}}$

$$
a b=a \cdot b+a \wedge b
$$

that is, the geometric product is a linear combination of the internal and external product. It can be seen how the result is made up of a scalar and a bivector, resulting in the so-called multivector.

$$
\boldsymbol{A}=\boldsymbol{a} \boldsymbol{b}=\langle\boldsymbol{A}\rangle_{0}+\langle\boldsymbol{A}\rangle_{2}=\left(\alpha_{1} \alpha_{2}+\beta_{1} \beta_{2}\right)+\left(\alpha_{1} \beta_{2}-\beta_{1} \alpha_{2}\right) \boldsymbol{e}_{\mathbf{1}} \boldsymbol{e}_{\mathbf{2}}
$$

$\langle\boldsymbol{A}\rangle_{0}$ is the scalar part and $\langle\boldsymbol{A}\rangle_{2}$ is the bivector part. A bivector is a novel concept introduced by geometric algebra and does not exist in vector calculus. It is the result of the external product of two vectors producing a plane with direction and sense, exactly as a vector would have it. Its value is equal to the area enclosed by the parallelogram formed by the vectors (see Figure 2). Like vectors, a bivector can be written as the linear combination of a base of bivectors. 
Based on the transformation given by Castro-Nuñez [16]

$$
\begin{array}{llc}
\varphi_{c 1}(t)=\sqrt{2} \cos \omega t & e_{1} \\
\varphi_{s 1}(t)=\sqrt{2} \sin \omega t & -e_{2} \\
\varphi_{c 2}(t)=\sqrt{2} \cos 2 \omega t \longleftrightarrow e_{2} e_{3} \\
\varphi_{s 2}(t)=\sqrt{2} \sin 2 \omega t \longleftrightarrow e_{1} e_{3} \\
\varphi_{c n}(t)=\sqrt{2} \cos n \omega t \longleftrightarrow \bigwedge_{i=2}^{n+1} e_{i} \\
\varphi_{s n}(t)=\sqrt{2} \sin n \omega t \longleftrightarrow \bigwedge_{\substack{i=1 \\
i \neq 2}}^{n+1} e_{i} \\
\end{array}
$$

we can transform the Fourier spectrum to GA domain, $\mathcal{G}_{\mathcal{N}}$. For example, consider the voltage waveform

$$
u(t)=230 \sqrt{2} \sin \omega t+110 \sqrt{2} \sin 3 \omega t
$$

following the proposed transformation in (6), the voltage becomes

$$
\boldsymbol{u}=\underbrace{-230 \boldsymbol{e}_{\mathbf{2}}}_{\langle\boldsymbol{u}\rangle_{1}}+\underbrace{110 \boldsymbol{e}_{\mathbf{1 3 4}}}_{\langle\boldsymbol{u}\rangle_{3}}
$$

In equation (6), every integer multiple of sine and cosine has a unique translation to a base k-vector, but there is no correspondence for interharmonics components, i.e., there is no way to transform interharmonics from the frequency domain to GA. One of the main contribution of this paper is to provide new rules to complete the geometric algebra theory applied to electrical circuits. Let us suppose we have an interharmonic $u_{\text {int }}(t)=U \sqrt{2} \cos h \omega t$, where $1<h<2$, i.e., $h$ is between fundamental component and $2^{\text {nd }}$ harmonic and 
it is clear that $h \notin \mathbb{N}$. The transformation is as follows

$$
\cos (h \omega t) \longleftrightarrow e_{24}
$$

If other interharmonics exists in the interval $[1,2]$, then terms like $\boldsymbol{e}_{\mathbf{2 5}}, \boldsymbol{e}_{\mathbf{2 6}}$, etc will arise. In general, if $\mathrm{k}$ interharmonics, $p_{k}$, exist between $n$ and $n+1$, the transformation is as follows

$$
\begin{aligned}
X_{c_{n} p_{k}} & =\left(\bigwedge_{i=2}^{n+1} e_{i}\right) e_{(k+n+2)} \\
X_{s_{n} p_{k}} & =\left(\bigwedge_{\substack{i=1 \\
i \neq 2}}^{n+1} e_{i}\right) e_{(k+n+2)}
\end{aligned}
$$

where subscript $c$ means cosine and $s$ means sine transformation, respectively.

Once voltage and current can be represented in the geometrical domain, it is possible to define a new power concept called geometrical apparent power or net apparent power $\boldsymbol{M}$, as it is described by CastroNúñez [23]. This concept supersedes the traditional concept of apparent power $S$ widely known in electrical engineering. This change is both substantive and profound, but it is supported by numerous studies that demonstrate how traditional apparent power is unable to explain with clarity and coherence the flow of energy and power in non-linear systems, where harmonics and interharmonics appear [24]. The term reactive power is also misinterpreted in its conceptual meaning of quadrature power with active power [25, 26]. These terms work very well in linear and sinusoidal systems, but they founder when applied to non-sinusoidal or non-linear systems. It is precisely $\boldsymbol{M}$ that comes to overcome this lack, guaranteeing the principle of energy conservation and a physical correspondence between the different terms of which it is composed and the characteristics of the problem. One of the consequences of the foregoing is that, in general, $\|M\| \neq S$. The geometric apparent power is defined as the geometric product between voltage and current:

$$
M=u i=u \cdot i+u \wedge i
$$

which will result in a scalar and a bivector for the sinusoidal case

$$
\boldsymbol{M}=\underbrace{\langle\boldsymbol{M}\rangle_{0}}_{\text {scalar }}+\underbrace{\langle\boldsymbol{M}\rangle_{2}}_{\text {bivector }}
$$

It can be generalized for a non-sinusoidal voltage with the presence of interharmonics 


$$
\begin{aligned}
u(t)=\sum_{i=1}^{n} u_{i}(t) & =D_{1} \cos (\omega t)+E_{1} \sin (\omega t)+\sum_{h=2}^{d} D_{h} \cos (h \omega t)+\sum_{h=2}^{k} E_{h} \sin (h \omega t)+ \\
& +\sum_{p \in J} P_{p} \sin (p \omega t)
\end{aligned}
$$

where $n$ is the total number of frequency components of the signal, $d$ and $k$ are the total number of harmonics and $J$ is the set of interharmonics. After that, it can be obtained the voltage transferred to the geometric domain $\mathcal{G}_{\mathcal{N}}$

$$
\begin{aligned}
\boldsymbol{u}= & D_{1} \boldsymbol{e}_{\mathbf{1}}-E_{1} \boldsymbol{e}_{\mathbf{2}}+\sum_{h=2}^{d}\left[D_{h} \bigwedge_{i=2}^{h+1} \boldsymbol{e}_{\boldsymbol{i}}\right]+\sum_{h=2}^{k}\left[E_{h} \bigwedge_{i=1, i \neq 2}^{h+1} \boldsymbol{e}_{\boldsymbol{i}}\right]+ \\
& +\sum_{p_{p} \in J_{1}}\left[\bigwedge_{\substack{i=1 \\
i \neq 2}}^{n+1} \boldsymbol{e}_{\boldsymbol{i}}\right] \boldsymbol{e}_{(\boldsymbol{p}+\boldsymbol{n}+\mathbf{2})}+\sum_{p_{p} \in J_{2}}\left[\bigwedge_{i=2}^{n+1} \boldsymbol{e}_{\boldsymbol{i}}\right] \boldsymbol{e}_{(\boldsymbol{p}+\boldsymbol{n}+\mathbf{2})}
\end{aligned}
$$

Since Castro-Nuñez [23] establishes that the geometric admittance is $\boldsymbol{Y}=G_{h}+B_{h} \boldsymbol{e}_{\mathbf{1}} \boldsymbol{e}_{\mathbf{2}}$, applying the superposition principle (SP) yield each of the harmonic currents as $\boldsymbol{i}_{\boldsymbol{h}}=\left(G_{h}+B_{h} \boldsymbol{e}_{\mathbf{1}} \boldsymbol{e}_{\mathbf{2}}\right) \boldsymbol{u}_{\boldsymbol{h}}$. Clearly, the total current $\boldsymbol{i}$ is the sum of all harmonic currents

$$
\boldsymbol{i}=\sum_{h=1}^{n} \boldsymbol{i}_{\boldsymbol{h}}
$$

This current can be decomposed into parallel and quadrature components with the voltage

$$
i=i_{\|}+i_{\perp}=i_{g}+i_{b}
$$

Finally, the apparent multivector geometric power $\boldsymbol{M}$ can be obtained as the product between $\boldsymbol{u}$ and $\boldsymbol{i}$ 


$$
\begin{aligned}
M & =u i=M_{g}+M_{b}=\underbrace{\overbrace{\left\langle M_{g}\right\rangle_{0}+\sum_{i=1}^{n+1}\left\langle M_{g}\right\rangle_{i}}^{P}}_{M_{g}}+ \\
& +\underbrace{C N_{r(p s)}+C N_{r(h i)}}_{M_{b}=C N_{r}}
\end{aligned}
$$

where

$\boldsymbol{M}_{\boldsymbol{g}}$ is the parallel geometric apparent power

$\boldsymbol{M}_{\boldsymbol{b}}$ is the cuadrature geometric apparent power

$\boldsymbol{P}$ is the active power

$\boldsymbol{C} \boldsymbol{N}_{\boldsymbol{d}}$ is the degraded power

$C N_{r}$ is the geometric reactive power

$C \boldsymbol{N}_{r(p s)}$ is the geometric reactive power due to voltage and current phase shift

$C \boldsymbol{N}_{\boldsymbol{r}(\boldsymbol{h} i)}$ is the geometric reactive power due to voltage and current cross products

Based on the above definitions, the net or geometric power factor can be defined as

$$
p f=\frac{P}{\|\boldsymbol{M}\|}=\frac{\langle\boldsymbol{M}\rangle_{0}}{\sqrt{\left\langle\boldsymbol{M}^{\dagger} \boldsymbol{M}\right\rangle_{0}}}
$$

\section{Empirical study}

To demonstrate the validity and usefulness of the proposed method for the resolution of non-sinusoidal and non-linear electric circuits based on geometric algebra, several examples of typical circuits will be solved. 


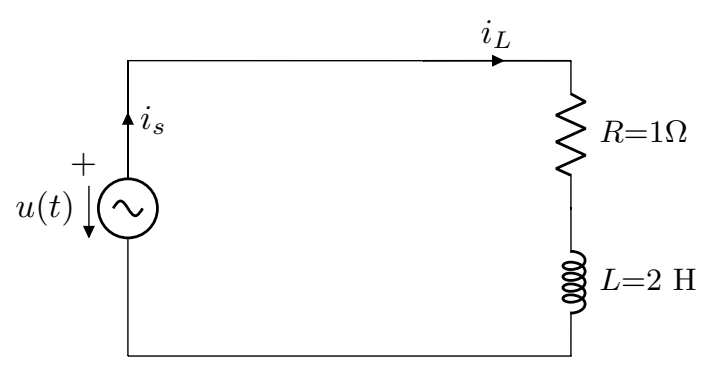

Figure 3: Linear inductive load

\subsection{Example 1}

The first case of study (Figure 3) represents a simple inductive linear load powered by a non-sinusoidal voltage source polluted with interharmonic and harmonic

$$
v(t)=230 \sqrt{2} \sin \omega t+20 \sqrt{2} \sin 1.2 \omega t+10 \sqrt{2} \sin 3 \omega t
$$

for the sake of simplicity, $\omega=1$ will be taken.

The voltage waveform is transformed into geometric domain using (6), so that the geometric voltage is as follows

$$
u=-230 e_{2}+20 e_{14}+10 e_{134}
$$

The geometric impedance $\boldsymbol{z}_{\boldsymbol{h}}$ for each frequency component is defined (according to what is proposed in [23], as:

$$
z_{h}=R-h \omega L e_{12}
$$

where $h$ is the order of the harmonic or interharmonic, in our problem, $h=[1,1.2,3]$.

$$
\begin{aligned}
& z_{1}=1-2 e_{12} \\
& z_{1.2}=1-2.4 e_{12} \\
& z_{3}=1-6 e_{12}
\end{aligned}
$$


Therefore, if we apply SP and Ohm's law, it is posible to calculate the current $\boldsymbol{i}$ as follows

$$
\begin{aligned}
\boldsymbol{i} & =\boldsymbol{z}_{\mathbf{1}}^{-1}\langle\boldsymbol{u}\rangle_{1}+\boldsymbol{z}_{\mathbf{1 . 2}}^{-1}\langle\boldsymbol{u}\rangle_{1.2}+\boldsymbol{z}_{\mathbf{3}}^{-1}\langle\boldsymbol{u}\rangle_{3} \\
& =\left(1-2 \boldsymbol{e}_{\mathbf{1 2}}\right)^{-1}\left(-230 \boldsymbol{e}_{\mathbf{2}}\right)+\left(1-2.4 \boldsymbol{e}_{\mathbf{1 2}}\right)^{-1}\left(20 \boldsymbol{e}_{\mathbf{1 4}}\right)+\left(1-6 \boldsymbol{e}_{\mathbf{1 2}}\right)^{-1}\left(10 \boldsymbol{e}_{\mathbf{1 3 4}}\right) \\
& =\underbrace{-46 \boldsymbol{e}_{\mathbf{2}}+2.96 \boldsymbol{e}_{\mathbf{1 4}}+0.27 \boldsymbol{e}_{\mathbf{1 3 4}}}_{\boldsymbol{i}_{\boldsymbol{g}}} \underbrace{-92 \boldsymbol{e}_{\mathbf{1}}-7.1 \boldsymbol{e}_{\mathbf{2 4}}-1.62 \boldsymbol{e}_{\mathbf{2 3 4}}}_{\boldsymbol{i}_{\boldsymbol{b}}}
\end{aligned}
$$

Note that the principle of superposition is embedded in equation (22) and consequently all the harmonics (interharmonics) can be examined simultaneously. This is in contrast to the frequency domain, where it is no possible to handle all harmonics at once. The value of the total current is found by its norm $\|\boldsymbol{i}\|=103.16 \mathrm{~A}$. It should be noted that the current obtained has two distinct components: $\boldsymbol{i}_{\boldsymbol{g}}$ is the parallel current and $\boldsymbol{i}_{\boldsymbol{b}}$ is the quadrature current. It's easy to verify that both components are in quadrature simply by performing the scalar product $\boldsymbol{i}_{\boldsymbol{g}} \cdot \boldsymbol{i}_{\boldsymbol{b}}=0$. Once the current is determined, the geometric apparent power $\boldsymbol{M}$ can be calculated

$$
\boldsymbol{M}=\boldsymbol{u} \boldsymbol{i}=\underbrace{10641+35 \boldsymbol{e}_{\mathbf{3}}+1600 \boldsymbol{e}_{\mathbf{1 2 4}}-398 \boldsymbol{e}_{\mathbf{1 2 3 4}}}_{\boldsymbol{M}_{\boldsymbol{g}}}+\underbrace{3473 \boldsymbol{e}_{\mathbf{4}}-21000 \boldsymbol{e}_{\mathbf{1 2}}-547 \boldsymbol{e}_{\mathbf{3 4}}-103 \boldsymbol{e}_{\mathbf{1 2 3}}}_{M_{b}}
$$

so that the detailed analysis of powers appears as

$$
\begin{gathered}
P=10,641 \mathrm{~W} \\
\left\|\boldsymbol{C} \boldsymbol{N}_{\boldsymbol{d}}\right\|=1,650 \mathrm{VA} \\
\left\|\boldsymbol{C} \boldsymbol{N}_{\boldsymbol{r}}\right\|=21,294 \mathrm{VA} \\
\|\boldsymbol{M}\|=23,862 \mathrm{VA}
\end{gathered}
$$

It is easy to demonstrate that

$$
\|M\|=\sqrt{P^{2}+\left\|C N_{d}\right\|^{2}+\left\|C N_{r}\right\|^{2}}
$$

The power factor is then obtained by applying (18)

$$
p f=\frac{10,641}{23,862}=0.446
$$




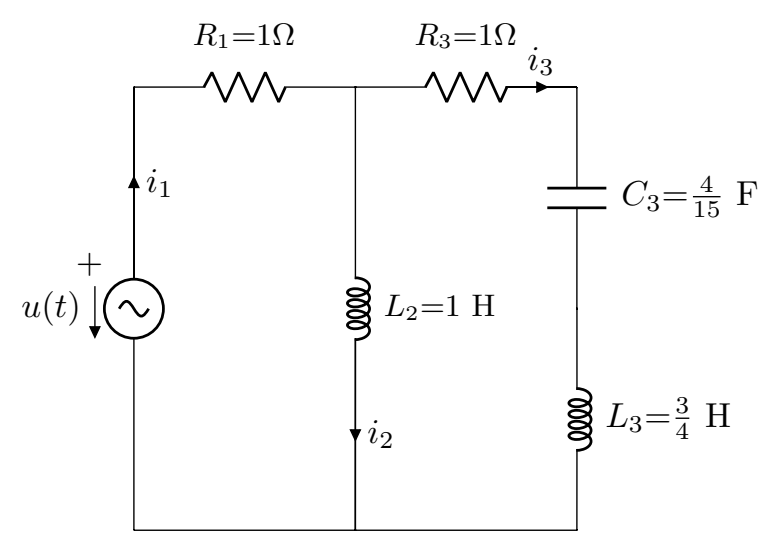

Figure 4: Linear circuit for example 2.

\begin{tabular}{|c|c|c|c|c|}
\hline \multirow[b]{2}{*}{ Element } & \multicolumn{2}{|c|}{$h=1$} & \multicolumn{2}{|c|}{$h=1.7$} \\
\hline & Impedance $\boldsymbol{Z}$ & Admittance $\boldsymbol{Y}$ & Impedance $\boldsymbol{Z}$ & Admittance $\boldsymbol{Y}$ \\
\hline$R_{1}$ & 1 & 1 & 1 & 1 \\
\hline$L_{2}$ & $-e_{12}$ & $e_{12}$ & $-1.7 e_{12}$ & $0.588 e_{12}$ \\
\hline$R_{3}$ & 1 & 1 & 1 & 1 \\
\hline$L_{3}$ & $-0.75 e_{12}$ & $1.333 \boldsymbol{e}_{12}$ & $-1.275 e_{12}$ & $0.784 \boldsymbol{e}_{12}$ \\
\hline$C_{3}$ & $3.75 \boldsymbol{e}_{12}$ & $-0.266 e_{12}$ & $2.206 \boldsymbol{e}_{\mathbf{1 2}}$ & $-0.453 e_{12}$ \\
\hline$R L C_{3}$ & $1+3 \boldsymbol{e}_{\mathbf{1 2}}$ & $0.1-0.3 \boldsymbol{e}_{12}$ & $1+0.93 \boldsymbol{e}_{\mathbf{1 2}}$ & $0.536-0.498 \boldsymbol{e}_{\mathbf{1 2}}$ \\
\hline$R L C_{123}$ & $1.2-1.4 e_{12}$ & $0.353+0.412 \boldsymbol{e}_{\mathbf{1 2}}$ & $2.81-0.30 \boldsymbol{e}_{12}$ & $0.352+0.037 e_{12}$ \\
\hline
\end{tabular}

Table 1: Geometric impedance and admittance for circuit elements

\subsection{Example 2}

The second case of study is showed in Figure 4. In this case, a more complex linear circuit is presented with non-sinusoidal source and serveral resistors, inductors and capacitors.

The voltage source is

$$
v(t)=230 \sqrt{2} \sin \omega t+23 \sqrt{2} \sin 1.7 \omega t
$$

which translates to geometric domain as

$$
\boldsymbol{u}=-230 \boldsymbol{e}_{\mathbf{2}}+23 \boldsymbol{e}_{14}
$$




\begin{tabular}{|c|c|c|c|c|c|}
\hline & \multicolumn{5}{|c|}{ k-vector } \\
\hline & $e_{1}$ & $e_{2}$ & $e_{14}$ & $e_{24}$ & $\|\boldsymbol{i}\|$ \\
\hline$i_{2}$ & $-148,8235$ & $-94,7059$ & 0,5118 & $-8,7798$ & 176,6210 \\
\hline$i_{3}$ & 54,1176 & 13,5294 & 7,5626 & 7,9098 & 56,8465 \\
\hline$i_{1}$ & $-94,7059$ & $-81,1765$ & 8,0743 & $-0,8700$ & 125,0000 \\
\hline
\end{tabular}

Table 2: Current summary in amperes $[\mathrm{A}]$

Similar to the example 1 above, the geometric impedance can be calculated for every element in figure 4 as showed in table 1 .The geometric current is derived by applying Ohm's law for every branch $m=1,2,3$

$$
\boldsymbol{i}_{m}=\sum_{k} \boldsymbol{z}_{\boldsymbol{k m}}^{-1}\langle\boldsymbol{u}\rangle_{k m}
$$

The table 2 shows a summary of the three current branches of the circuit $\boldsymbol{i}_{\mathbf{1}}, \boldsymbol{i}_{\mathbf{2}}$ and $\boldsymbol{i}_{\mathbf{3}}$. It can be observed that $\boldsymbol{i}_{1}=\boldsymbol{i}_{2}+\boldsymbol{i}_{3}$ as stated by Kirchhoff's 1st law. Recovering equation (16), the current $\boldsymbol{i}_{\mathbf{1}}$ can be decomposed as a parallel and quadrature parts

$$
\boldsymbol{i}_{1}=\boldsymbol{i}_{\boldsymbol{g} \mathbf{1}}+\boldsymbol{i}_{\boldsymbol{b} \mathbf{1}}=\underbrace{-81.17 \boldsymbol{e}_{\mathbf{2}}+8.07 \boldsymbol{e}_{14}}_{\boldsymbol{i}_{\boldsymbol{g} 1}} \underbrace{-94.70 \boldsymbol{e}_{1}-0.87 \boldsymbol{e}_{\mathbf{2}}}_{\boldsymbol{i}_{\boldsymbol{b} 1}}
$$

The quadrature current $\boldsymbol{i}_{\boldsymbol{b} \mathbf{1}}$ is the part that can be compensated by passive filters using techniques such heuristic optimization provided in [21].

The apparent geometrical power involved can be found by the geometric product between voltage and current in each passive element. Table 3 shows the power balance detail for each element of the circuit in figure 4. It is clear that the principle of energy conservation is fulfilled as presented in [23].

Finally, in order to compare the GA power theory applied to non-sinusoidal and non-linear systems, the time domain power of the source $p(t)$ has been calculated. The voltage $v(t)$ and current $i_{1}(t)$ is multiplied to find the instantaneous power as 


\begin{tabular}{|c|c|c|c|c|c|}
\hline \multirow[b]{3}{*}{$M_{R 1}$} & \multicolumn{5}{|c|}{ k-vector } \\
\hline & $e_{0}$ & $e_{4}$ & $e_{12}$ & $e_{124}$ & $\|M\|$ \\
\hline & $15,624.77$ & & & 1475.67 & $15,694.30$ \\
\hline$M_{L 2}$ & & 3658.79 & $-30,986.15$ & 528.73 & $31,205.90$ \\
\hline$M_{R 3}$ & 3231.52 & & & 651.48 & 3296.54 \\
\hline$M_{L 3}$ & & 659.63 & -2181.13 & 271.04 & 2294.75 \\
\hline$M_{C 3}$ & & -1940.09 & $11,404.94$ & 797.20 & $11,596.21$ \\
\hline$M_{3}$ & 3231.52 & -1280.46 & 9223.81 & 1719.74 & $10,005.92$ \\
\hline$M$ & $18,856.29$ & 2378.33 & $-21,762.34$ & 3724.15 & $29,132.20$ \\
\hline
\end{tabular}

Table 3: Geometric apparent power summary. Active power P in watts [W] and the rest of terms in volt-amperes [VA].

$$
\begin{aligned}
& p(t)=[\underbrace{230 \sin \omega t+23 \sin 1.7 \omega t}_{u(t)}][\underbrace{-94.70 \cos \omega t+81.17 \sin \omega t+8.07 \sin 1.7 \omega t-0.87 \cos 1.7 \omega t}_{i_{1}(t)}] \\
& =\underbrace{\underbrace{18670.6}_{P_{1}} \underbrace{(\sin \omega t)^{2}}_{\left(-e_{2}\right)^{2}}+\underbrace{185.7}_{P_{1.7}} \underbrace{(\sin 1.7 \omega t)^{2}}_{\left(e_{14}\right)^{2}}}_{P}-\underbrace{21782.4}_{C N_{r(p s)}} \underbrace{\sin \omega t \cos \omega t}_{C N_{r 1}}-\underbrace{20.0}_{-e_{21}=e_{12}} \underbrace{\sin 1.7 \omega t \cos 1.7 \omega t}_{C N_{r 1.7}} \\
& -\underbrace{200.1 \underbrace{\sin \omega t \cos 1.7 \omega t}_{-e_{4}}-2178.2 \underbrace{\sin 1.7 \omega t \cos \omega t}_{-e_{4}}}_{C N_{r(h i)}}+\underbrace{3724.1}_{C N_{d}} \underbrace{\sin 1.7 \omega t \sin \omega t}_{\mathbb{N}_{124}}
\end{aligned}
$$

In the above equation it can be clearly seen how the different terms of the instantaneous power have an evident translation in the terms of the geometric apparent power $M$. For the sake of clarity, different colors have been used to highlight each of these components. For example, the active power $\mathrm{P}$ is the sum of the squared sine terms that arise when multiplying the same harmonic voltage and current order. Other cross terms are also related by the degraded power $\boldsymbol{C} \boldsymbol{N}_{\boldsymbol{d}}$ or the power in quadrature due to the interaction between harmonics of different order.

\subsection{Example 3}

The third example is a non-linear load connected to a real voltage source according to the diagram shown in Figure 5. The problem data are as follows: 


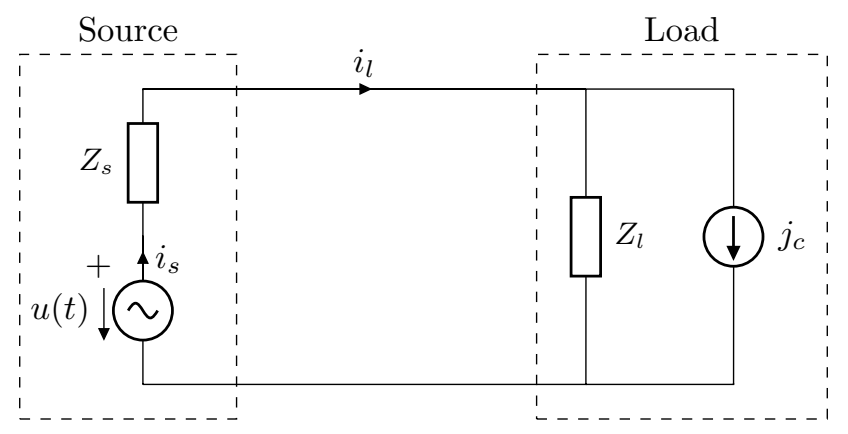

Figure 5: Non-linear load and real voltage source

$$
\begin{aligned}
u(t) & =230 \sqrt{2} \sin \omega t+10 \sqrt{2} \sin 3 \omega t+5 \sqrt{2} \sin 3.5 \omega t \\
j_{c}(t) & =10 \sqrt{2} \sin 3 \omega t+\sqrt{2} \sin 4.5 \omega t \\
R_{s} & =R_{l}=1 \Omega \\
L_{s} & =L_{l}=1 \mathrm{H}
\end{aligned}
$$

Following the steps of the previous examples, we proceed to carry out the transformation from the time domain to the geometric domain, so that

$$
\begin{aligned}
\boldsymbol{u} & =-230 \boldsymbol{e}_{\mathbf{2}}+10 \boldsymbol{e}_{\mathbf{1 3 4}}+5 \boldsymbol{e}_{\mathbf{1 3 4 6}} \\
\boldsymbol{j}_{\boldsymbol{c}} & =10 \boldsymbol{e}_{\mathbf{1 3 4}}+\boldsymbol{e}_{\mathbf{1 3 4 5 7}} \\
\boldsymbol{z}_{\boldsymbol{h}} & =R-h \omega L \boldsymbol{e}_{\mathbf{1 2}}
\end{aligned}
$$

After that, the principle of superposition and Ohm's law is applied to solve the different currents present in the circuit, i.e. $\boldsymbol{i}_{\boldsymbol{s}}$ and $\boldsymbol{i}_{\boldsymbol{l}}$. To do this, some operations are performed in the geometric domain taking into account that $\boldsymbol{i}_{\boldsymbol{x}}=\left\langle i_{x}\right\rangle_{1}+\left\langle i_{x}\right\rangle_{3}+\left\langle i_{x}\right\rangle_{3.5}+\left\langle i_{x}\right\rangle_{4.5}$,

$$
\begin{aligned}
\boldsymbol{i}_{\boldsymbol{l}} & =\left[\boldsymbol{z}_{\mathbf{1} \boldsymbol{l}}\right]^{-1}\left\langle\boldsymbol{u}_{\boldsymbol{l}}\right\rangle_{1}+\left[\boldsymbol{z}_{\mathbf{3} \boldsymbol{l}}\right]^{-1}\left\langle\boldsymbol{u}_{\boldsymbol{l}}\right\rangle_{3}+\left[\boldsymbol{z}_{\mathbf{3 . 5} \boldsymbol{l}}\right]^{-1}\left\langle\boldsymbol{u}_{\boldsymbol{l}}\right\rangle_{3.5}+\left[\boldsymbol{z}_{\mathbf{4 . 5} \boldsymbol{l}}\right]^{-1}\left\langle\boldsymbol{u}_{\boldsymbol{l}}\right\rangle_{4.5} \\
\boldsymbol{u}_{\boldsymbol{l}} & =\left\langle\boldsymbol{u}_{\boldsymbol{l}}\right\rangle_{1}+\left\langle\boldsymbol{u}_{\boldsymbol{l}}\right\rangle_{3}+\left\langle\boldsymbol{u}_{\boldsymbol{l}}\right\rangle_{3.5}+\left\langle\boldsymbol{u}_{\boldsymbol{l}}\right\rangle_{4.5}
\end{aligned}
$$


For a better understanding of the solving process, a step-by-step approach will be made, although all these steps can be carried out simultaneously. First, the voltage source will be solved and then the current source, following the superposition principle.

(i) Voltage source

When only the voltage source is applied, the equivalent circuit is

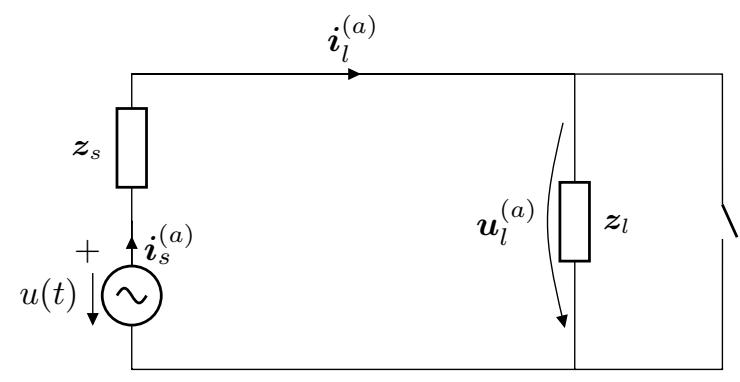

Solving the voltage $\boldsymbol{u}_{l}^{(a)}$ and the current $\boldsymbol{i}_{l}^{(a)}$ for each frequency component

$$
\begin{aligned}
\boldsymbol{u}_{l}^{(a)} & =\boldsymbol{z}_{l 1}\left\langle\boldsymbol{i}_{s}^{(a)}\right\rangle_{1}+\boldsymbol{z}_{l 3}\left\langle\boldsymbol{i}_{l}^{(a)}\right\rangle_{3}+\boldsymbol{z}_{l 3.5}\left\langle\boldsymbol{i}_{l}^{(a)}\right\rangle_{3.5}=\left(1-\boldsymbol{e}_{\mathbf{1 2}}\right) \frac{-230 \boldsymbol{e}_{\mathbf{2}}}{\left(1-\boldsymbol{e}_{\mathbf{1 2}}\right)+\left(1-\boldsymbol{e}_{\mathbf{1 2}}\right)}+ \\
& +\left(1-3 \boldsymbol{e}_{\mathbf{1 2}}\right) \frac{10 \boldsymbol{e}_{\mathbf{1 3 4}}}{\left(1-3 \boldsymbol{e}_{\mathbf{1 2}}\right)+\left(1-3 \boldsymbol{e}_{\mathbf{1 2}}\right)}+\left(1-3.5 \boldsymbol{e}_{\mathbf{1 2}}\right) \frac{5 \boldsymbol{e}_{\mathbf{1 3 4 6}}}{\left(1-3.5 \boldsymbol{e}_{\mathbf{1 2}}\right)+\left(1-3.5 \boldsymbol{e}_{\mathbf{1 2}}\right)}= \\
& =-115 \boldsymbol{e}_{\mathbf{2}}+5 \boldsymbol{e}_{\mathbf{1 3 4}}+2.5 \boldsymbol{e}_{\mathbf{1 3 4 6}} \\
\boldsymbol{i}_{l}^{(a)}= & \frac{-230 \boldsymbol{e}_{\mathbf{2}}}{\left(1-\boldsymbol{e}_{\mathbf{1 2}}\right)+\left(1-\boldsymbol{e}_{\mathbf{1 2}}\right)}+\frac{10 \boldsymbol{e}_{\mathbf{1 3 4}}}{\left(1-3 \boldsymbol{e}_{\mathbf{1 2}}\right)+\left(1-3 \boldsymbol{e}_{\mathbf{1 2}}\right)}+\frac{5 \boldsymbol{e}_{\mathbf{1 3 4 6}}}{\left(1-3.5 \boldsymbol{e}_{\mathbf{1 2}}\right)+\left(1-3.5 \boldsymbol{e}_{\mathbf{1 2}}\right)}= \\
= & -57.5 \boldsymbol{e}_{\mathbf{1}}-57.5 \boldsymbol{e}_{\mathbf{2}}+0.5 \boldsymbol{e}_{\mathbf{1 3 4}}-1.5 \boldsymbol{e}_{\mathbf{2 3 4}}+0.19 \boldsymbol{e}_{\mathbf{1 3 4 6}}-0.66 \boldsymbol{e}_{\mathbf{2 3 4 6}}
\end{aligned}
$$

(ii) Current source

If we only apply the current source, the circuit to operate on is 


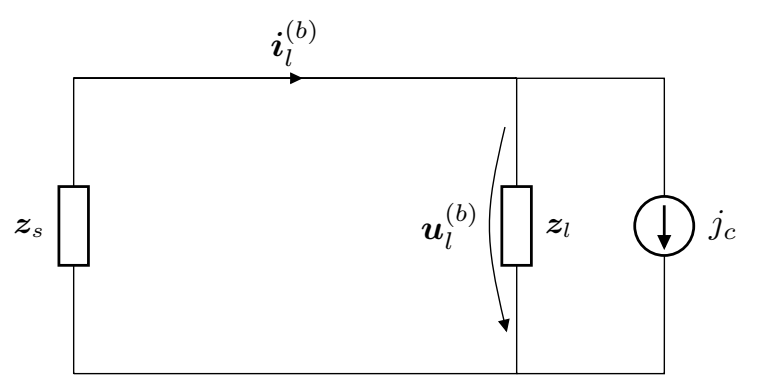

that helps us to solve the voltage $\boldsymbol{u}_{l}^{(b)}$

$$
\begin{gathered}
\boldsymbol{u}_{l}^{(b)}=-\frac{1}{2}\left(1-3 \boldsymbol{e}_{\mathbf{1 2}}\right)\left\langle\boldsymbol{j}_{c}\right\rangle_{3}-\frac{1}{2}\left(1-4.5 \boldsymbol{e}_{\mathbf{1 2}}\right)\left\langle\boldsymbol{j}_{c}\right\rangle_{4.5}= \\
=-\frac{1}{2}\left(1-3 \boldsymbol{e}_{\mathbf{1 2}}\right) 10 \boldsymbol{e}_{\mathbf{1 3 4}}-\frac{1}{2}\left(1-4.5 \boldsymbol{e}_{\mathbf{1 2}}\right) \boldsymbol{e}_{\mathbf{1 3 4 5 7}}= \\
=-5 \boldsymbol{e}_{\mathbf{1 3 4}}-15 \boldsymbol{e}_{\mathbf{2 3 4}}-0.5 \boldsymbol{e}_{\mathbf{1 3 4 5 7}}-2.25 \boldsymbol{e}_{\mathbf{2 3 4 5 7}} \\
\boldsymbol{i}_{l}^{(b)}=5 \boldsymbol{e}_{\mathbf{1 3 4}}+0.5 \boldsymbol{e}_{\mathbf{1 3 4 5 7}}
\end{gathered}
$$

The total load voltage will be

$$
\boldsymbol{u}_{l}=\boldsymbol{u}_{l}^{(a)}+\boldsymbol{u}_{l}^{(b)}=-115 \boldsymbol{e}_{\mathbf{2}}-15 \boldsymbol{e}_{\mathbf{2 3 4}}+2.5 \boldsymbol{e}_{\mathbf{1 3 4 6}}-0.5 \boldsymbol{e}_{\mathbf{1 3 4 5 7}}-2.25 \boldsymbol{e}_{\mathbf{2 3 4 5 7}}
$$

and the total current demanded by the load

$$
\boldsymbol{i}_{l}=\boldsymbol{i}_{l}^{(a)}+\boldsymbol{i}_{l}^{(b)}=-57.5 \boldsymbol{e}_{\mathbf{1}}-57.5 \boldsymbol{e}_{\mathbf{2}}+5.5 \boldsymbol{e}_{\mathbf{1 3 4}}-1.5 \boldsymbol{e}_{\mathbf{2 3 4}}+0.19 \boldsymbol{e}_{\mathbf{1 3 4 6}}-0.66 \boldsymbol{e}_{\mathbf{2 3 4 6}}+0.5 \boldsymbol{e}_{\mathbf{1 3 4 5 7}}
$$

Finally, the geometric apparent power is equal to the geometric product between voltage and current 


$$
\begin{aligned}
\boldsymbol{M}=\boldsymbol{u i} & =6635.22+3.85 \boldsymbol{e}_{\mathbf{6}} \\
& -6695.52 \boldsymbol{e}_{\mathbf{1 2}}+1,035 \boldsymbol{e}_{\mathbf{3 4}}-0.62 \boldsymbol{e}_{\mathbf{5 7}} \\
& -6.60 \boldsymbol{e}_{\mathbf{1 2 6}}+219.65 \boldsymbol{e}_{\mathbf{3 4 6}}+0.14 \boldsymbol{e}_{\mathbf{5 6 7}} \\
& -230 \boldsymbol{e}_{\mathbf{1 2 3 4}}-20.62 \boldsymbol{e}_{\mathbf{1 2 5 7}}+158.12 \boldsymbol{e}_{\mathbf{3 4 5 7}} \\
& +165.60 \boldsymbol{e}_{\mathbf{1 2 3 4 6}}+0.75 \boldsymbol{e}_{\mathbf{1 2 5 6 7}} \\
& -43.12 \boldsymbol{e}_{\mathbf{1 2 3 4 5 7}}
\end{aligned}
$$

The absolute value of the geometric power is

$$
\|M\|=9491.22 \mathrm{VA}
$$

and the power factor

$$
p f=\frac{6635}{9491.22}=0.7
$$

As in the previous examples, geometrical power $\boldsymbol{M}$ can be decomposed into different constituents such as $\boldsymbol{M}_{\boldsymbol{g}}$ or $\boldsymbol{M}_{\boldsymbol{b}}$, i.e. components due to parallel current or quadrature with voltage, respectively. It is interesting to remark that the use of geometric algebra complies with the principle of energy conservation by faithfully reflecting the power flows involved due to the different spectral components. For example, the active power $P$ is worth $6635.22 \mathrm{~W}$ and is due to the interaction of similar components between voltage and current harmonics,

$$
\begin{aligned}
P & =\left\langle M_{h 1}\right\rangle_{0}+\left\langle M_{h 3}\right\rangle_{0}+\left\langle M_{h 3.5}\right\rangle_{0}+\left\langle M_{h 4.5}\right\rangle_{0} \\
& =6612.5+22.5+0.475-0.25=6635.22 \mathrm{~W}
\end{aligned}
$$

So, as expected, the total active power is the sum of every harmonic interaction taking into account the sense of the energy flow. Only the interharmonic 4.5 is flowing in the opposite direction, i.e., from load to source. 


\section{Conclusions}

In this paper we present an extension of Castro-Nuñez's theory for the analysis of electric circuits by means of geometric algebra. Through the use of GA, voltage and current harmonics and interharmonics can be handled simultaneously, in such a way that it is possible to solve any type of electric circuit, whether it is linear or non-linear, including harmonics and interharmonics. This technique exceeds by far the use of complex numbers traditionally employed by electrical engineers. It is also proven how the geometrical product of voltage and current leads to several power constituents that make it possible to verify the principle of energy conservation, a fact that is not possible with the traditional concepts of apparent and reactive power. The examples proposed and solved validate the use of GA for dealing with the interaction of power terms coming from cross products of harmonics, interharmonics and fundamental component, resulting in new components such as the degraded power $C N_{d}$ and the quadrature power $C N_{r}$. This contribution opens a fundamentally new avenue for the study of power systems with non-linear loads and non-sinusoidal sources.

\section{References}

[1] M. H. Bollen, Understanding power quality problems, in: Voltage sags and Interruptions, IEEE press, 2000.

[2] E. Viciana, A. Alcayde, F. Montoya, R. Baños, F. Arrabal-Campos, A. Zapata-Sierra, F. Manzano-Agugliaro, Openzmeter: An efficient low-cost energy smart meter and power quality analyzer, Sustainability 10 (11) (2018) 4038.

[3] E. W. Gunther, Interharmonics in power systems, in: 2001 Power Engineering Society Summer Meeting. Conference Proceedings (Cat. No. 01CH37262), Vol. 2, IEEE, 2001, pp. 813-817.

[4] C. Li, W. Xu, T. Tayjasanant, Interharmonics: basic concepts and techniques for their detection and measurement, Electric Power Systems Research 66 (1) (2003) 39-48.

[5] F. Montoya, R. Baños, A. Alcayde, M. Montoya, F. Manzano-Agugliaro, Power quality: Scientific collaboration networks and research trends, Energies 11 (8) (2018) 2067.

[6] L. Czarnecki, Non-periodic currents: their properties, identification and compensation fundamentals, in: 2000 Power Engineering Society Summer Meeting (Cat. No. 00CH37134), Vol. 2, IEEE, 2000, pp. 971-976.

[7] L. Czarnecki, Circuits with semi-periodic currents: Main features and power properties, European transactions on electrical power 12 (1) (2002) 41-46.

[8] L. Feola, R. Langella, A. Testa, On the effects of interharmonic distortion on measurement instruments based on pll systems, in: 2012 IEEE International Workshop on Applied Measurements for Power Systems (AMPS) Proceedings, IEEE, 2012, pp. 1-6.

[9] V. Ravindran, S. K. Rönnberg, T. Busatto, M. H. Bollen, Inspection of interharmonic emissions from a grid-tied pv inverter in north sweden, in: 2018 18th International Conference on Harmonics and Quality of Power (ICHQP), IEEE, 2018, pp. 1-6.

[10] T. Kim, E. J. Powers, W. M. Grady, A. Arapostathis, Real and reactive power analysis for interharmonics, in: IEEE Electric Ship Technologies Symposium, 2005., IEEE, 2005, pp. 244-247. 
[11] W.-K. Yoon, M. J. Devaney, Reactive power measurement using the wavelet transform, IEEE Transactions on instrumentation and measurement 49 (2) (2000) 246-252.

[12] P. Petrovic, M. Stevanovic, Measuring active power of synchronously sampled ac signals in presence of interharmonics and subharmonics, IEE Proceedings-Electric Power Applications 153 (2) (2006) 227-235.

[13] D. Hestenes, G. Sobczyk, Clifford algebra to geometric calculus: a unified language for mathematics and physics, Vol. 5, Springer Science \& Business Media, 2012.

[14] D. Hestenes, New foundations for classical mechanics, Vol. 15, Springer Science \& Business Media, 2012.

[15] M. Castro-Nuñez, R. Castro-Puche, The ieee standard 1459, the cpc power theory, and geometric algebra in circuits with nonsinusoidal sources and linear loads, IEEE Transactions on Circuits and Systems I: Regular Papers 59 (12) (2012) 2980-2990.

[16] M. Castro-Nuñez, R. Castro-Puche, Advantages of geometric algebra over complex numbers in the analysis of networks with nonsinusoidal sources and linear loads, IEEE Transactions on Circuits and Systems I: Regular Papers 59 (9) (2012) 2056-2064.

[17] A. Menti, T. Zacharias, J. Milias-Argitis, Geometric algebra: a powerful tool for representing power under nonsinusoidal conditions, IEEE Transactions on Circuits and Systems I: Regular Papers 54 (3) (2007) 601-609.

[18] M. Castilla, J. C. Bravo, M. Ordoñez, Geometric algebra: a multivectorial proof of tellegen's theorem in multiterminal networks, IET circuits, devices \& systems 2 (4) (2008) 383-390.

[19] M. Castilla, J. C. Bravo, M. Ordonez, J. C. Montaño, Clifford theory: a geometrical interpretation of multivectorial apparent power, IEEE Transactions on Circuits and Systems I: Regular Papers 55 (10) (2008) 3358-3367.

[20] M. Castro-Núñez, D. Londoño-Monsalve, R. Castro-Puche, M, the conservative power quantity based on the flow of energy, The Journal of Engineering 2016 (7) (2016) 269-276.

[21] F. G. Montoya, A. Alcayde, F. M. Arrabal-Campos, R. Baños, Quadrature current compensation in non-sinusoidal circuits using geometric algebra and evolutionary algorithms, Energies 12 (4) (2019) 692.

[22] J. M. Chappell, S. P. Drake, C. L. Seidel, L. J. Gunn, A. Iqbal, A. Allison, D. Abbott, Geometric algebra for electrical and electronic engineers, Proceedings of the IEEE 102 (9) (2014) 1340-1363.

[23] M. Castro-Núñez, R. Castro-Puche, E. Nowicki, The use of geometric algebra in circuit analysis and its impact on the definition of power, in: Nonsinusoidal Currents and Compensation (ISNCC), 2010 International School on, IEEE, 2010, pp. 89-95.

[24] L. S. Czarnecki, On some misinterpretations of the instantaneous reactive power pq theory, IEEE transactions on power electronics 19 (3) (2004) 828-836.

[25] L. S. Czarnecki, What is wrong with the budeanu concept of reactive and distortion power and why it should be abandoned, IEEE Transactions on Instrumentation and measurement 1001 (3) (1987) 834-837.

[26] J. L. Willems, Budeanu's reactive power and related concepts revisited, IEEE Transactions on Instrumentation and Measurement 60 (4) (2011) 1182-1186. 